\title{
Estimulação com marca-passo diafragmático em lesão medular cervical alta: relato de caso
}

\author{
Camila S. Monteiro, ${ }^{1}$ Victor Emmanuel C. Zamora, ${ }^{1 *}$ Ana Carolina A. Carvalho, ${ }^{1}$ Sergio Cunha ${ }^{1}$
}

\begin{abstract}
Resumo
O objetivo deste relato de caso foi estabelecer protocolo de estimulação via marca-passo diafragmático, pela equipe de fisioterapia do Hospital Universitário Pedro Ernesto, visando desmame da ventilação mecânica invasiva de uma paciente com lesão medular alta internada na Unidade de Terapia Intensiva há 13 anos por dependência de ventilação artificial. Verificou-se aumento do volume corrente durante a estimulação e também aumento em cinco vezes na pressão inspiratória máxima comparado ao valor inicial. Entretanto, o treino foi interrompido no $40^{\circ}$ dia devido à instalação de neuromielite óptica que a fez perder condução dos nervos frênicos momentaneamente.
\end{abstract}

Descritores: Marca-passo diafragmático; Nervo frênico; Lesão medular cervical; Desmame do respirador; Ventilação mecânica invasiva; Fisioterapia respiratória.

\section{Abstract \\ Stimulation diaphragmatic pacemaker for high cervical spinal cord injury: a case report}

The objective of this case report is to set a protocol for the stimulation via an artificial diaphragmatic pacemaker, by the physiotherapy team at the Pedro Ernesto University Hospital, aiming the weaning from the invasive mechanical ventilation of a marrow damaged patient admitted to the ICU for 13 years due to the need of assisted ventilation. It has been identified an increase of the tidal volume during the stimulation and of 5 times of the maximum inspiratory pressure (PImax) compared to the initial rate. However, the procedure was interrupted in the 40th day due to an optical neuromyelitis that led to a temporary loss of the phrenic nerve conduction.

Keywords: Diaphragmatic pacemaker; phrenic nerve; Cervical cord damage; Ventilatory weaning; Invasive mechanical ventilation; Respiratory physiotherapy.
1. Unidade de Terapia Intensiva. Hospital Universitário Pedro Ernesto. Universidade do Estado do Rio de Janeiro. Rio de Janeiro, RJ, Brasil.

*Endereço para correspondência:

Setor de Fisioterapia, HUPE

Boulevard 28 de Setembro, 77, 40 andar

Rio de Janeiro, RJ. Brasil. CEP: 20550-030.

E-mail: fisiovictor.br@gmail.com

Revista HUPE, Rio de Janeiro, 2016;15(1):82-85

doi: 10.12957/rhupe.2016.22379

Recebido em 18/10/2015. Aprovado em 20/02/2016.

\section{Resumen}

Estimulación con marcapasos diafragmático en lesiones de la médula cervical alta: relato de un caso

El propósito de este relato de caso fue establecer el protocolo de estimulación a través de marcapasos diafragmático, el equipo de fisioterapia, buscando el destete de la ventilación mecánica invasiva en una paciente con lesión medular alta, internada en la Unidad de Terapia Intensiva hace 13 años por dependencia de ventilación artificial. Se verificó aumento del volumen de corriente durante la estimulación y también aumento de cinco veces en la presión inspiratoria máxima, en comparación con el valor inicial. Sin embargo, la práctica se interrumpió después de 40 días debido a la neuromielitis óptica que hizo perder la conducción de los nervios frénico momentáneamente.

Palabras clave: Marcapasos diafragmático; Nervio frénico; Lesión medular cervical; Destete del respirador; Ventilación mecánica invasiva; Fisioterapia respiratoria. 


\section{Caso clínico}

\section{Introdução}

A lesão medular cervical alta, geralmente causa dependência de ventilação mecânica invasiva (VMI), uma vez que o diafragma recebe inervação primariamente do nível C4 com contribuição de C3 e C5. ${ }^{1}$

A dependência de VMI pode gerar algumas complicações como: infecções das vias aéreas superiores e pneumonia associada à ventilação mecânica, condições que podem reduzir a expectativa de vida. ${ }^{2}$ Essas complicações podem ser evitadas com o uso do marca-passo diafragmático, permitindo também a fonação, redução de custos e melhora da qualidade de vida. ${ }^{3}$

As principais indicações para o implante do marca-passo diafragmático são: pacientes com lesão medular cervical alta e a síndrome da hipoventilação central congênita. ${ }^{4}$ Como critério de seleção para o implante, $\mathrm{o}$ paciente deve apresentar função preservada dos nervos frênicos e do músculo diafragma. 25,6

O marca-passo diafragmático (MPD) pode ser um estimulador implantável no nervo frênico, tanto na porção cervical quanto na torácica, que proporciona contração diafragmática,7 ou um estimulador implantável diretamente no diafragma. .

O sistema do MPD de nervos frênicos consiste em eletrodos suturados nesses nervos, conectados a receptores implantados em cavidades subcutâneas, que recebem informação do transmissor externo Mark IV por meio de antenas externamente acopladas aos receptores. ${ }^{8}$

O MPD geralmente é ativado quatro semanas após a cirurgia, sendo o programa de condicionamento diafragmático realizado com ativação deste, sem auxílio de VMI. ${ }^{9} \mathrm{O}$ condicionamento requer um aumento gradual do tempo de estimulação, fazendo-se importante a monitorização de volume corrente $(\mathrm{VC})$ através da ventilometria. ${ }^{8,10}$

\section{Relato do caso}

Paciente com 30 anos de idade, sexo feminino, diagnóstico de meningoencefalite e mielite transversa provocando lesão completa em medula cervical alta, que se encontra internada na UTI do Hospital Universitário Pedro Ernesto (HUPE), Rio de Janeiro.

Aos 17 anos de idade, adquiriu otite, evoluindo com meningite e abscesso em fossa posterior do crânio. Sofreu abordagem cirúrgica sem resolução do quadro, ocasionando, portanto, tetraplegia espástica e dependência de VMI. Nesse período, foi admitida na UTI do referido hospital, onde permanece internada apresentando espasticidade grau 2 e grau 3, em membros inferiores e membros superiores, respectivamente, pela escala modificada de Ashworth, ausência de função motora e sensitiva abaixo do nível de lesão. Não apresenta úlceras de pressão e possui função cognitiva preservada, alimentando-se por via oral apesar de traqueostomizada. Além disso, recebe aulas de alfabetização e pintura com a boca por professoras voluntárias e atualmente participa de uma associação internacional de pintores com a boca.

Após 13 anos de lesão, em abril de 2013 foi realizado implante do MPD por estimulação bilateral do nervo frênico nível cervical por cervicotomia pela equipe do núcleo de implantação de MPD do estado do Rio de Janeiro no HUPE, composto por profissionais de saúde desse hospital. O desmame da VMI foi iniciado 28 dias após a implantação, sem auxílio de VMI durante a mesma.

Algumas condutas precediam o treinamento, como: higiene brônquica e oxigenação de 1 minuto com $\mathrm{FiO}_{2}$ a $100 \%$. A paciente era posicionada em decúbito dorsal, com cabeceira elevada a $30-45^{\circ}$. Além disso, utilizamos uma faixa elástica abdominal a fim de fornecer o apoio biomecânico adequado, melhorando assim a zona de aposição do diafragma e minimizando prejuízos mecânicos causados pela flacidez dos músculos abdominais.

Ao iniciar o condicionamento, foi necessário ajustar a frequência respiratória (FR) do transmissor e a amplitude de estímulo de cada nervo. Determinamos uma FR de duas incursões abaixo das que a paciente realizava na VMI a fim de evitar possível fadiga por excesso de estímulo. A amplitude (em volts) de estímulo do MPD varia de 1 a $9 \mathrm{v}$; para determiná-la, verificamos o limiar ascendente, ou seja, a amplitude que gera maior VC na ventilometria. Foi verificada a amplitude de cada lado separadamente. Determinamos FR=16 irpm, amplitude de melhor VC à esquerda $=3 \mathrm{v}$ e amplitude de melhor VC à direita $=8 \mathrm{v}$.

Durante a estimulação foi realizada monitorização contínua dos sinais vitais incluindo $\mathrm{SpO}_{2}$, ventilometria através do ventilômetro de Wright (Nspire ${ }^{\circledR}$, Alemanha) e sensação subjetiva de esforço através da escala modificada de Borg. A ventilometria foi realizada no primeiro minuto, na metade do período e no último minuto de estimulação. A monitorização tem a finalidade de evidenciar qualquer alteração hemodinâmica e/ou redução $\geq 50 \%$ do VC aferido, o que indicaria fadiga diafragmática?

No primeiro dia de treino foi realizada estimulação 
com MPD, sem auxílio de VMI, por 5 minutos, 5 vezes ao dia, com pelo menos 2 horas de descanso entre as estimulações. $\mathrm{O}$ repouso entre os treinos foi mantido com o ventilador mecânico Servo -S (Maquet, Suécia), modo ventilatório assisto/controlado, controlado à pressão, com pressão inspiratória de $14 \mathrm{cmH}_{2} \mathrm{O}$, pressão expiratória final positiva (PEEP) de $8 \mathrm{cmH}_{2} \mathrm{O}$ e $\mathrm{FiO}_{2}$ a $21 \%$. No segundo dia, ao progredir para 10 minutos, a paciente apresentou redução significativa do VC. Desse modo, foi mantido o tempo de estimulação de 5 minutos durante 1 semana, com a finalidade de uma melhor adaptação diafragmática ao estímulo.

Após esses dias de adaptação, foi realizada uma progressão diária de 5 minutos de estimulação até atingir 50 minutos contínuos de treino. A partir deste momento, a progressão passou a ser de 10 minutos por dia.

A cada 3 dias, realizamos ventilometria e manovacuometria sem o MPD, a fim de mensurar volume minuto (VM), VC e força muscular respiratória.

A paciente realizou 40 dias de condicionamento muscular diafragmático com o MPD, apresentando aumento gradativo de $\mathrm{VC}$ a cada treino, com progressões de 5-10 minutos diários no tempo de estimulação. Essa resposta satisfatória foi observada até o $24^{\circ}$ dia de treinamento.

No $25^{\circ}$ dia de estimulação, apresentou fadiga do hemidiafragma direito, constatada por redução superior a 50\% do VC aferido anteriormente e pela ausência de contração visível e palpável desse músculo. Oferecemos, portanto, repouso de 72 horas, retomando o treinamento com metade do tempo de estimulação em que houve a falha.

Seguindo o plano de treinamento, no $40^{\circ}$ dia a paciente completou 2 horas e 50 minutos de estimulação diafragmática contínua e independente de VMI. No entanto, nesse mesmo dia, apresentou sinais de fadiga ventilatória, sendo o treino interrompido.

Nos dias subsequentes, tentou-se retomar a estimulação, com constantes falhas; investigou-se mais profundamente o motivo destas, verificando-se que a paciente havia desenvolvido neuromielite óptica, ocasionando perda da condução dos nervos frênicos demonstrada na eletroneuromiografia, não sendo possível prosseguir com o desmame.

Observamos aumento progressivo do VC no decorrer dos dias de estimulação, exceto no $25^{\circ}$ e $40^{\circ}$ dia, quando houve fadiga (Gráfico 1).

Apesar de o condicionamento ter sido interrompido, obtivemos resultados satisfatórios após 40 dias de estimulação. O VC inicial aumentou de $240 \mathrm{ml}$ para
$709 \mathrm{ml}$ ao final do treinamento, sem haver incremento da amplitude de estímulo, demonstrando melhora do condicionamento muscular diafragmático.

Observou-se melhora da força muscular tanto inspiratória quanto expiratória, sendo a primeira mais evidente. A paciente apresentou uma melhora expressiva da PImáx, de $-8 \mathrm{cmH}_{2} \mathrm{O}$ antes do treinamento para $-40 \mathrm{cmH}_{2} \mathrm{O}$. A PEmáx evoluiu de $+10 \mathrm{cmH}_{2} \mathrm{O}$ para +20 $\mathrm{cmH}_{2} \mathrm{O}$ ao final do período (Gráficos 2 e 3 ).

\section{Discussão}

A progressão do tempo de estimulação ainda não está bem estabelecida na literatura. Autores sugerem para pacientes tetraplégicos, um aumento de 3-5 minutos por dia; ${ }^{11}$ realizamos neste estudo um treinamento inicial de 5 minutos com aumento gradativo de $5 \mathrm{mi}-$ nutos diários objetivando evitar fadiga por excesso de estimulação, por se tratar de uma paciente tetraplégica há 13 anos, com provável atrofia difusa do diafragma.

Em nosso estudo, optamos por utilizar uma cinta abdominal durante a estimulação com MPD a fim de melhorar a zona de aposição do diafragma, otimizando a mecânica ventilatória da paciente. Observamos um aumento no VC em torno de $150 \mathrm{ml}$ ao utilizarmos a cinta. Alguns autores já comprovaram que a perda de volume pulmonar está associada com a perda da força muscular abdominal, e os benefícios de usar uma cinta abdominal em pacientes tetraplégicos para auxiliar a ventilação estão descritos na literatura. ${ }^{12}$

Alguns autores descreveram o relato de um paciente dependente de VMI há 12 anos após fratura de C1-C2 que atingiu 10 horas de independência de VMI com MPD. ${ }^{12}$

Durante a estimulação de longa duração do nervo frênico, as fibras rápidas são convertidas em fibras resistentes à fadiga por estimulação de baixa frequência, além disso, a força e a resistência do diafragma aumentam com a estimulação. ${ }^{20}$ Observamos em nosso relato de caso um aumento tanto da força muscular inspiratória, visualizada por expressiva melhora da PImáx, estando 5 vezes melhor comparado ao valor inicial, quanto melhora da resistência diafragmática com MPD, expressa pelo aumento do VC mesmo com progressões no tempo de estimulação, demonstrando que, apesar de longo período de internação associada à VMI prolongada, o diafragma dessa paciente ainda possui certo grau de plasticidade comprovando a efetividade do condicionamento diafragmático. Não se sabe se esse efeito poderá ser encontrado em outros pacientes com mesmo quadro clínico. 


\section{Caso clínico}

Gráfico 1. Progressão do volume corrente durante o período de treinamento com marca-passo diafragmático.

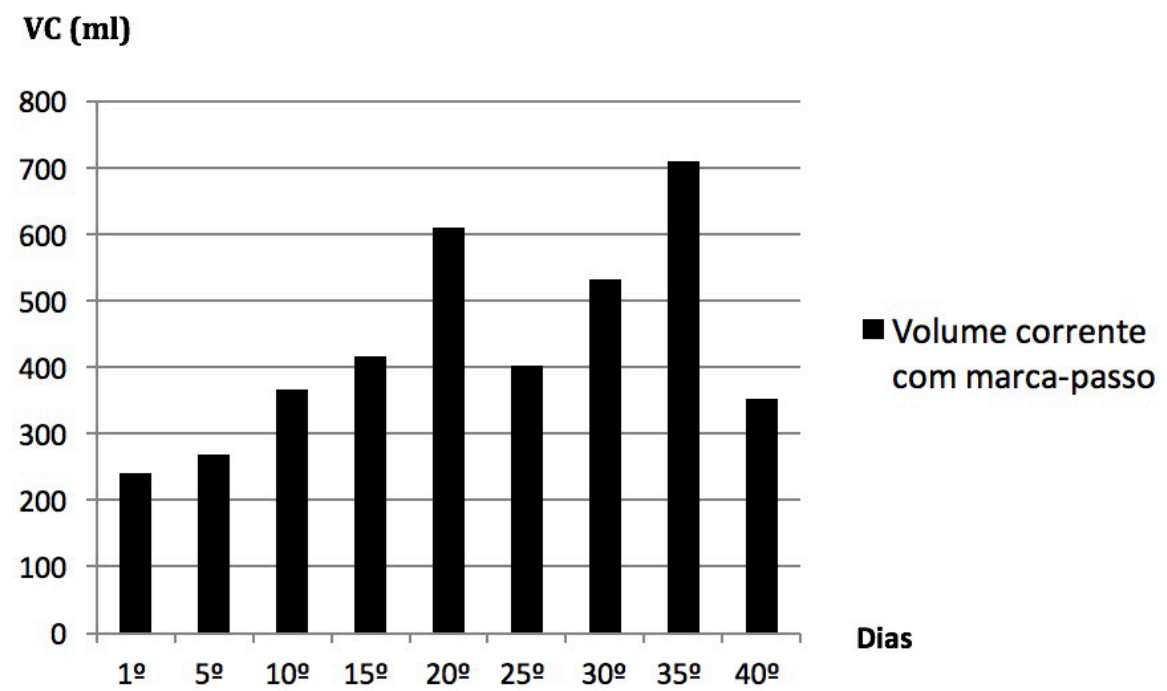

Legenda: VC (ml): volume corrente em mililitros; 1 a 40 dias de treinamento com MPD.

Gráfico 2. Pressão Inspiratória e Expiratória Máximas no decorrer dos 40 dias de Treinamento Muscular com Marca-passo Diafragmático.

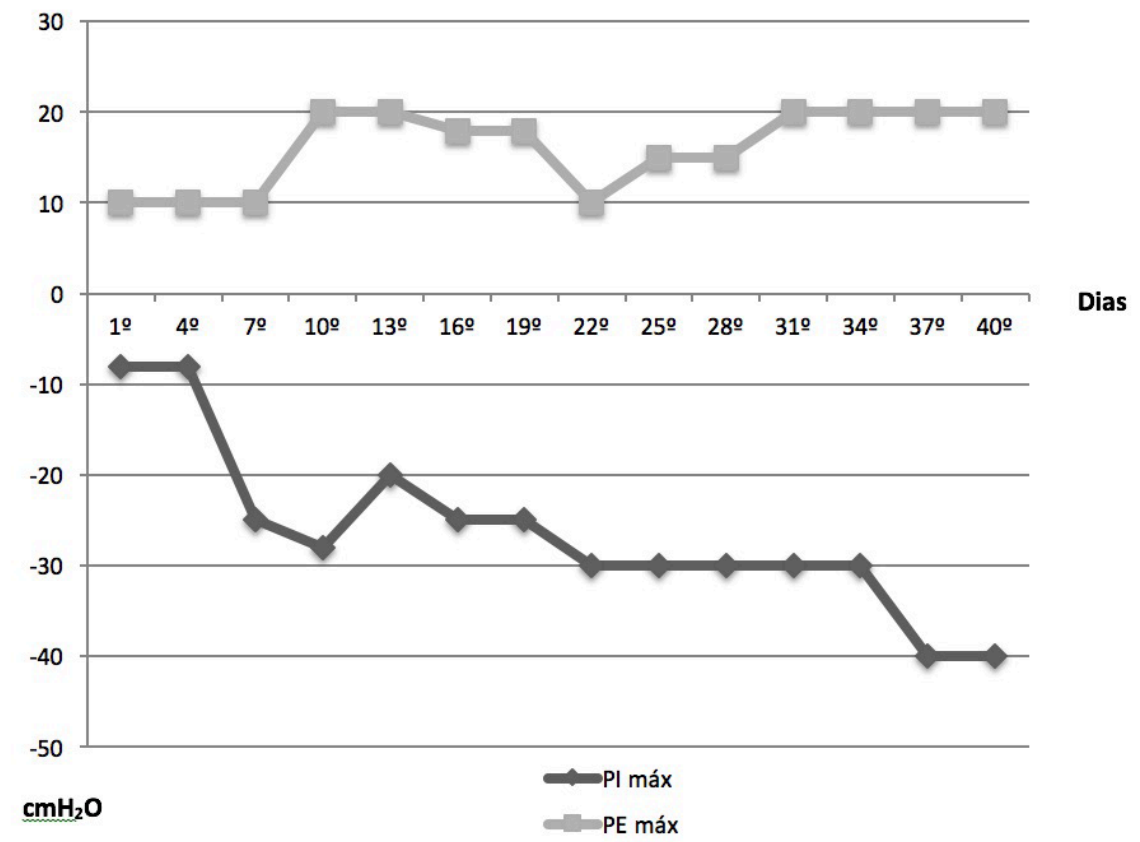

Legenda: PI máx: pressão inspiratória máxima em centímetros de água; PE máx: pressão expiratória máxima em centímetros de água. 1 a 40 dias de treinamento com MPD.

Apesar de o desmame ventilatório da paciente relatada não ter sido concluído em virtude do surgimento de uma nova afecção, a neuromielite óptica, verificamos evolução satisfatória com o protocolo utilizado até $\mathrm{o} 40^{\circ}$ dia de estimulação com MPD.
Sugere-se que o condicionamento muscular diafragmático com MPD seja uma alternativa viável para alcançar a ventilação independente, sendo capaz de aumentar tanto endurance quanto força do diafragma, mesmo em pacientes tetraplégicos em VMI prolongada. 
Gráfico 3. Pressão inspiratória e expiratória máximas antes e ao final dos 40 dias de treinamento muscular com marca-passo diafragmático

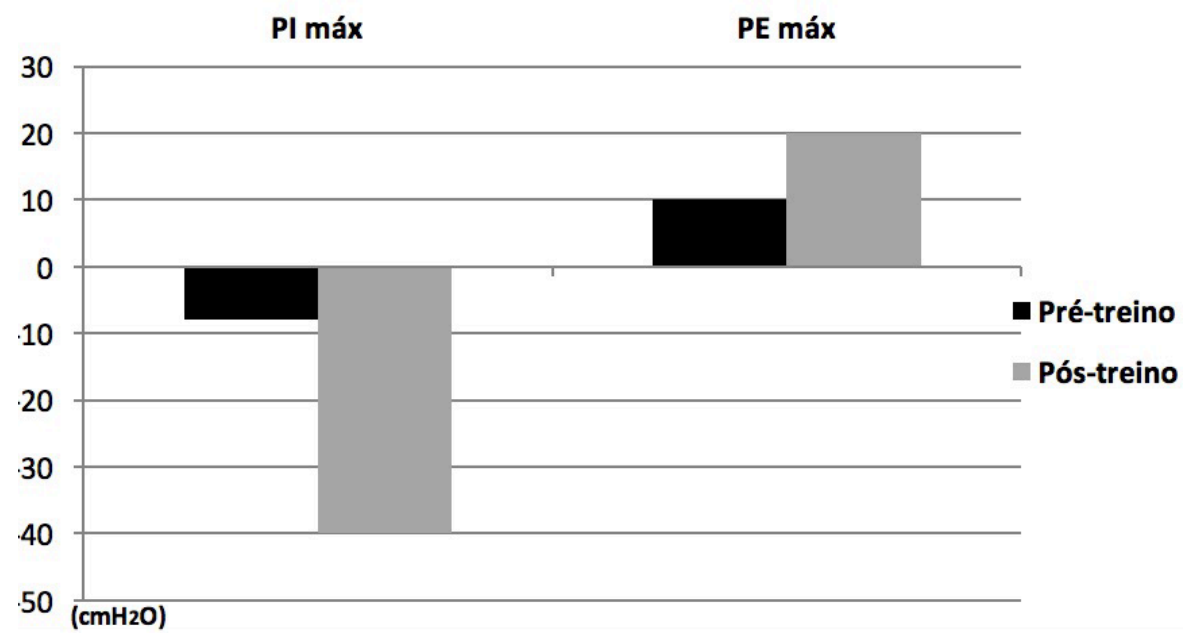

Legenda: PI máx: pressão inspiratória máxima em centímetros de água; PE máx: pressão expiratória máxima em centímetros de água.

\section{Referências}

1. Williams PL, Bannister LH, Berry MM. Gray’s Anatomy. 1995. New York: Churchill Livingstone.

2. Tedde ML, Onders RP, Teixeira MJ, et al. Ventilação elétrica: indicações e aspectos técnicos do implante cirúrgico do marca-passo de estimulação diafragmática. J Bras Pneumol. 2012; 38(5):566-572.

3. Hirschfeld S, Exner G, Luukkaala T, et al. Mechanical ventilation or phrenic nerve stimulation for treatment of spinal cord injury-induced respiratory insufficiency. Spinal Cord. 2008;46:738742.

4. Khong P, Lazzaro A. Phrenic nerve stimulation: The Australian experience. Journal of Clinical Neuroscience.2010;205-8.

5. Jarosz R, Littlepage MM, Creasey G, et al. Electrical stimulation to restore respiration. J Rehabil Res Dev. 1996;33:123-132.

6. Onders RP, Elmo M, Khansarinia S, et al. The learning curve for investigational surgery: lessons learned from laparoscopic diaphragm pacing for chronic ventilator dependence. Surg
Endosc. 2005;19(5):633-7.

7. Implanted breathing pacemaker system. Instruction manual. New York: Avery Biomedical Devices Inc. 2004.

8. Lam JCM, Ho CTK, Poon TL, et al. Implantation of a breathing pacemaker in a tetraplegic patient in Hong Kong. Hong Kong Med J. 2009;15:230-3.

9. McGee MF, Rosen MJ, Marks J, et al. Home-based ventilator weaning in tetraplegia: results of the diaphragm pacing stimulation system [abstract]. J Spinal Cord Med. 2006;29:249-250.

10. Miller JI, Farmer JA, Stuart W, et al. Phrenic nerve pacing of the quadriplegic patient. J Thorac Cardiovasc Surg. 1990;99(1):35-9;

11. Murray C, Seton C, Prelog K, et al. Arnold Chiari type 1 malformation presenting with sleep disordered breathing in well children. Arch Dis Child. 2006;91(4):342-3.

12. Talonen PP, Baer GA, Hakkinen V, et al. Neurophysiological and technical considerations for the design of an implantable phrenic nerve stimulation. Med Biol Eng Comput.1990;28:31-37. 